\title{
CONDUCTION PROCESSES IN THICK FILM RESISTORS PART II
}

\author{
M. P. ANSELL \\ Glass Division, Standard Telecommunication Laboratories Limited, London Road, Harlow, Essex, \\ England
}

(Received April 29, 1976)

\begin{abstract}
The structures of three families of thick film resistors have been investigated by scanning electron microscopy and electron probe micro-analysis. The two principal components of the resistive glazes, that is the conducting pigment and the glassy binder, have been identified in each case. The pigments were found to be simple or ternary oxides of the Pt transition metal group and Pd/PdO/Ag alloys. The glassy binders were based on lead borosilicate glasses.

A model for the electronic conduction processes through the glass and pigments is proposed on the basis of the observed physical structures, the measured electrical properties of resistors and the properties of the component resistor materials.

Part I of the total paper is concerned with identifying the phases in various thick film resistors and Part II considers the conduction processes that are applicable.
\end{abstract}

\section{INTRODUCTION}

Part II of this paper continues with a materials survey of the thick film resistor (TFR) phases identified in Part I. Conduction in multiphase materials is then considered, and low temperature coefficient of resistance (TCR) conduction processes are discussed. Finally conduction processes for the observed structures are proposed, and they are shown to be appropriate for TFRs with resistivities covering at least six orders of magnitude.

\section{TFR MATERIAL SURVEY}

Electron-probe micro-analysis of the principal conduction phases in the Du Pont 1100, Alloys B and Electroscience 7000 systems is consistent with the presence of $\mathrm{Bi}_{2} \mathrm{Ru}_{2} \mathrm{O}_{7}, \mathrm{RuO}_{2}$ and $\mathrm{Pd} / \mathrm{PdO} / \mathrm{Ag}$ alloys respectively. The matrix phases for these systems are standard or modified lead borosilicate glasses. The properties of these materials and their reported use in TFR systems are briefly reviewed.

\subsection{The Pd/PdO/Ag System}

Hoffmann ${ }^{1}$ describes a resistor system based on ballmilled particles of $\mathrm{Pd}$ and $\mathrm{Ag}$ from $0.1 \mu \mathrm{m}$ to $0.5 \mu \mathrm{m}$ in size, mixed with particles of a lead borosilicate glass. The aggregation of a $\mathrm{Pd} / \mathrm{Ag}$ alloy is said to form the conducting phases in the fired resistor. As Pd powder is heated in air it oxidises until a maximum oxygen content is reached ( $\mathrm{PdO})$. Above a certain temperature $\mathrm{PdO}$ begins to decompose, but small amounts of oxygen are left which raise the resistivity of the Pd. However the high energy exposed Pd surfaces react with $\mathrm{Ag}$ powder, sintering into phases of $\mathrm{Pd} / \mathrm{Ag}$ alloy solid solutions. These have a maximum resistivity of $56 \mathrm{wt} \% \mathrm{Pd}$ at which point the TCR is essentially zero-valued. This is possibly due to the completion of the Pd d-shell by a quantum mechanical sharing of $\mathrm{Ag}$ valence electrons, especially as the radius of the $\mathrm{Ag}$ ion is very similar to that of the $\mathrm{Pd}$ ion.

\subsection{The $\mathrm{RuO}_{2}$ System}

Angus and Gainsbury ${ }^{2}$ produced a series of resistors containing $\mathrm{RuO}_{2}$ because of its high metallic conductivity $\left(2.5 \times 10^{4} \Omega^{-1} \mathrm{~cm}^{-1}\right.$ at R.T. $)$ and chemical stability. By co-firing the oxide with a lead borosilicate glass a range of resistors from $10 \Omega$ /square to $10^{5} \Omega$ /square were produced with a $50 \%$ variation in conductor concentration, thus giving good control over resistivity. The electrical properties of Angus and Gainsbury's resistors are reproduced in Figure 1 for two ranges of particle sizes of $\mathrm{RuO}_{2}$.

Iles $^{3}$ and Lemon ${ }^{4}$ developed $\mathrm{RuO}_{2}$-glaze resistors incorporating niobium pentoxide. lles used up to 


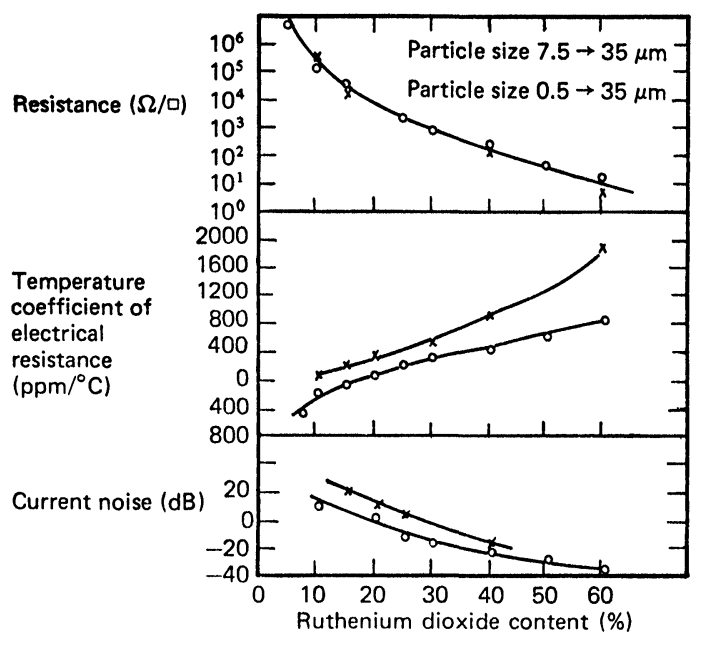

FIGURE 1 Electrical properties of ruthenium dioxide glaze resistors (Angus and Gainsbury [1968]).

$20 \mathrm{wt} \% \mathrm{Nb}_{2} \mathrm{O}_{5}$ calcined with the dioxide to control the TCR. $\mathrm{RuO}_{2}$ forms a solid solution with $\mathrm{Nb}_{2} \mathrm{O}_{5}$ and, on adding $20 \% \mathrm{Nb}_{2} \mathrm{O}_{5}$, the $\mathrm{RuO}_{2}$ lattice a parameter changes from $4.49 \AA$ to $4.57 \AA$ and the $\mathrm{c}$ parameter from $3.11 \AA$ to $3.07 \AA$. A 3.2 mole\% of $\mathrm{Nb}_{2} \mathrm{O}_{5}$ in $\mathrm{RuO}_{2}$ gives the alloy an effectively zero TCR over a wide range of resistor compositions. Mles states that up to $60 \mathrm{wt} \% \mathrm{Ag}$ can be used in place of $\mathrm{RuO}_{2}$ in resistor compositions without affecting the TCRs adversely. $\mathrm{Zn}$ and $\mathrm{Cd}$ borosilicates are found to be preferable to $\mathrm{Pb}$ borosilicates in that they can modify the TCRs of resistor compositions by $\pm 250 \mathrm{x}$ $10^{-6} \mathrm{~K}^{-1}$, which shows the importance of the glassy phase to the conduction process.

Van $\mathrm{Loan}^{5}$ reports that $\mathrm{RuO}_{2}$ is particularly com- patible with lead borosilicate glasses and that the oxide is insoluble in these glasses. Van Loan considers that conduction in $\mathrm{RuO}_{2}$ TFRs is along agglomerated particulate chains within the glass. He suggests that although $\mathrm{RuO}_{2}$ is insoluble in the glass, ions present in the glass could diffuse into the dioxide.

$\mathrm{RuO}_{2}$ crystallises in the rutile form of $\mathrm{TiO}_{2}$ with a tetragonal unit cell and 6:3 co-ordination. Resistivity versus temperture data for $\mathrm{RuO}_{2}$ is given by Ryden et $\mathrm{al}^{6}$, Rogers et $\mathrm{al}^{7}$ and Butler and Gillson ${ }^{8}$. The resistivity of single crystal $\mathrm{RuO}_{2}$ plotted logarithmically varies almost linearly with temperature from $10^{-8} \Omega \mathrm{cm}$ at $20 \mathrm{~K}$ to $10^{-4} \Omega \mathrm{cm}$ at $1000 \mathrm{~K}$. The majority of transition metal oxides are insulators but some are nearly degenerate semi-conductors. Some, such as $\mathrm{RuO}_{2}$, have metallic densities of electrons.

\subsection{The $\mathrm{Bi}_{2} \mathrm{Ru}_{2} \mathrm{O}_{7}$ System}

Bouchard ${ }^{9}$ describes electrically conducting bismuthcontaining oxides of pyrochlore-related crystal structure. Outstanding of these are the $\mathrm{Bi}_{2}(\mathrm{Ru}, \mathrm{Ir})_{2} \mathrm{O}_{7}$ series of compositions which have a virtually temperature-independent high conductivity. The resistivity of a sintered bar of $\mathrm{Bi}_{2} \mathrm{Ru}_{2} \mathrm{O}_{7}$ is $7 \times 10^{-4} \Omega$ $\mathrm{cm}$ at $4.2 \mathrm{~K}$ and $9 \times 10^{-4} \Omega \mathrm{cm}$ at $800 \mathrm{~K}$. Resistivity versus temperature data for three pyrochlore structure compounds are reproduced in Figure 2.

From the analysis of Coleman and Graves ${ }^{10}$ and this work it appears certain that $\mathrm{Bi}_{2} \mathrm{Ru}_{2} \mathrm{O}_{7}$ is a major phase in Du Pont 1100 resistors. Bouchard and coworkers ${ }^{11,12}$ have proposed that the metallic behaviour of $\mathrm{Bi}_{2}(\mathrm{Ru}, \mathrm{Ir})_{2} \mathrm{O}_{7}$ compounds depends on the partial filling of a conduction band formed by the overlap of electron orbitals. For metallic behaviour to occur a partially-filled conduction band must exist, but

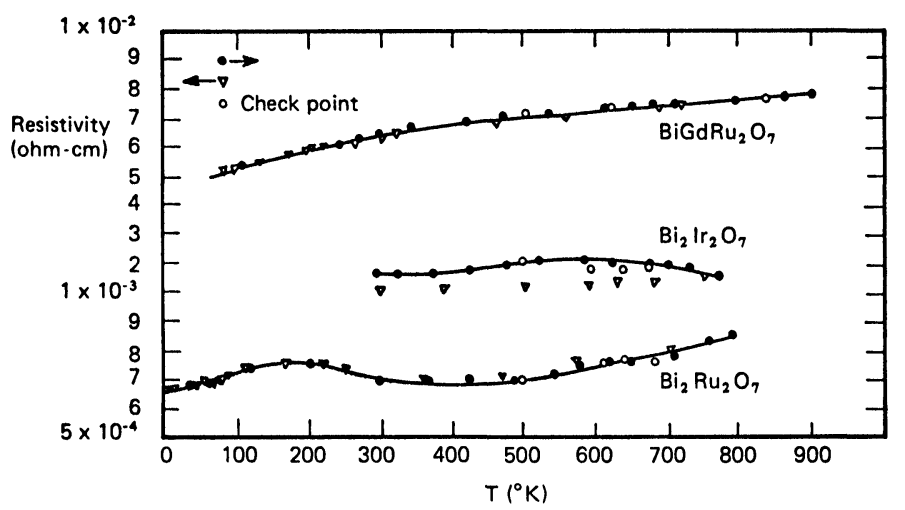

FIGURE 2 Resistivity versus temperature for three polycrystalline pyrochlores. Check points are made by cooling from some slightly higher temperature to assure that decomposition has not taken place. (Bouchard and Gillson [1971]). 
what is open to conjecture is the nature and width of the band. A low TCR material suggests a fixed mean free path and heavy scattering of large effective mass carriers. For carriers to have these properties they must either:

1) partially fill a narrow band and thus conduct metallically with a relatively high mobility, or

2) exist as a few states which require a very low energy for activation into a narrow band. $\mathrm{Tl}_{2} \mathrm{Ru}_{2} \mathrm{O}_{7}$, with $\mathrm{E}_{\mathrm{A}}=0.01 \mathrm{eV}$, is close to this interface between metals and semiconductors.

\subsection{Lead Borosilicate Glasses}

Glasses containing lead monoxide as a principal component have low melting temperatures, high resistance to devitrification, high surface and volume resistivities and high dielectric strength. By altering the basic components of lead glasses the thermal coefficient of expansion (TCE) can be easily controlled. These properties are particularly useful in TFR systems where the TCE of the conducting phase can be matched with the glass phase and substrate material, and the glass softening temperature, $\left(\mathrm{T}_{\mathrm{s}}\right)$, is reasonably low. Geller and Bunting ${ }^{13}$ measured $\mathrm{T}_{\mathrm{s}}$ and the TCE for the system $\mathrm{PbO} \cdot \mathrm{B}_{2} \mathrm{O}_{3} \cdot \mathrm{SiO}_{2}$. Glass compositions recorded in the TFR literature $2,9,14,15,16$ contain greater than $60 \% \mathrm{PbO}$, where compositions are highly stable and unreactive. The electrical properties of lead and lead-alumino borosilicates have also been investigated by Buchanan and Zuegel. ${ }^{17}$

\subsection{Interaction between Conductors and Glass}

During the resistor firing process structural re-ordering, chemical reaction and physical diffusion of the components may all occur. Solid solutions of glass and metal oxide may form and/or the glass may form a matrix around the oxide particles as seen in the micrographs.

$\mathrm{RuO}_{2}$ is stable up to $700^{\circ} \mathrm{C}$ and loses oxygen above this temperature. The dioxide is at least partially ionic and diffusion of $\mathrm{Ru}$ ions could take place under appropriate conditions of temperature and concentration gradients. Once $\mathrm{RuO}_{2}$ has been reduced to the metal it is very difficult to re-oxidise.

$\mathrm{Bi}_{2} \mathrm{Ru}_{2} \mathrm{O}_{7}$ is stable on heating in air up to at least $1000^{\circ} \mathrm{C}$ and is remarkably stable in reducing atmospheres.

Chemical action between substrate and resistor glass phase occurs at their interface probably due to alumina being a conditional glass-forming oxide.
However alumina substrates are inert up to very high temperatures so no reaction with the resistor bulk is likely. $\mathrm{RuO}_{2}$ and ruthenates are not glass-forming oxides and no reaction is expected with the substrate material.

Such reactions that do take place may be due to either chemical reactions or the formation of solid solutions between conductor and glass. Solid solution formation appears unlikely as Van Loan ${ }^{5}$ observed that the solubility of $15 \mathrm{wt} \%$ of $\mathrm{RuO}_{2}$ powder in an $85 \mathrm{wt} \% \mathrm{PbO} . \mathrm{B}_{2} \mathrm{O}_{3} \cdot \mathrm{SiO}_{2}$ glass phase was negligible. Bouchard" states that $\mathrm{Bi}_{2}(\mathrm{Ru}, \mathrm{Ir})_{2} \mathrm{O}_{7}$ compositions "are not adversely affected when heated with glass binders for TFR manufacture".

The TCE of substrates and glasses are well matched. The TCE of $\mathrm{RuO}_{2}\left(\sim 40 \times 10^{-7} \mathrm{~K}^{-1}\right)$ is less than the glass or substrate $\left(\sim 60\right.$ to $\left.80 \times 10^{-7} \mathrm{~K}^{-1}\right)$. However in the cooling part of the resistor firing cycle, the oxide component will be compressively locked into the glassy matrix. For low concentrations of oxide particles, expansion mismatches are easily absorbed whereas for high conductivity components the resistor structure should be physically weaker, which is the case.

\section{CONDUCTION IN COMPOSITE MATERIALS}

The preceding micrographs show the dispersed conducting oxide particles to form continuous particulate or filamentary chains. For conduction to occur through the high resistivity glassy phase it must either be predoped or doped during the firing process. The latter appears unlikely due to there being no reported reaction between glass and oxides. Alternatively hopping conduction between discrete oxide particles can occur through a thin glass film when the inter-particle spacing is less than $100 \AA^{18}$. If a single conduction process occurs it must be highly temperature independent. If several processes occur they must combine, over a suitable temperature range, to give almost temperature independent conductivity.

The Du Pont 1100 system $\left(\mathrm{Bi}_{2} \mathrm{Ru}_{2} \mathrm{O}_{7}\right)$ and the Electroscience 7000 system $(\mathrm{Pd} / \mathrm{PdO} / \mathrm{Ag})$ appear to have a single conduction process because of the already very low TCR of the conductor alone. Yet resistors covering six orders of magnitude of resistivity can be manufactured by simply adjusting the proportion of particles to glass. In any system where a distribution of conducting particles lies within a nonconducting matrix, a plot of log resistivity against particle concentration gives the general characteristic A of Figure 3, e.g. Malliaris and Turner ${ }^{19}$ for metal 


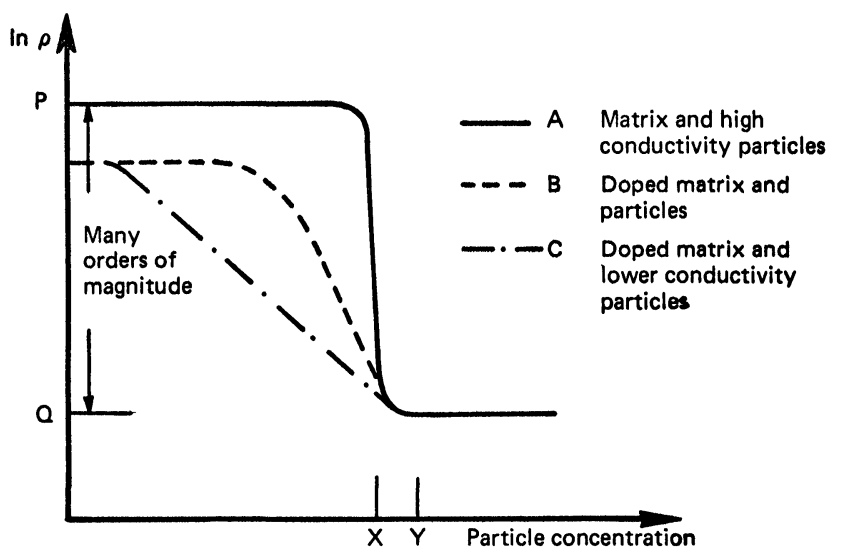

FIGURE 3 ln $\rho$ v. particle concentration for particles in a matrix

particles in plastic and Christen and Hewitt ${ }^{20}$ for $\mathrm{Au}-\mathrm{SiO}_{2}$ cermets.

$\mathrm{X}$ represents the approximate concentration at which particles begin to touch and $\mathrm{Y}$ represents the concentration at which all particles are in contact. Thus a small change in particle concentration, assuming that the particles are evenly dispersed, causes a change in resistivity from $P$, the resistance of the disordered matrix, to $\mathrm{Q}$, close to that of the bulk conductor. Thus intermediate values of resistivity will be difficult to obtain with any stability.

The characteristic A could be modified in two ways to increase the difference between concentration $\mathrm{X}$ and $\mathrm{Y}$. A dopant could be added to the disordered material which might provide trapping sites by affecting the balance of charge in the matrix of lower bulk conductivity. The general characteristic B in Figure 3 would then be expected. Conduction between trapping sites is necessarily a thermally-

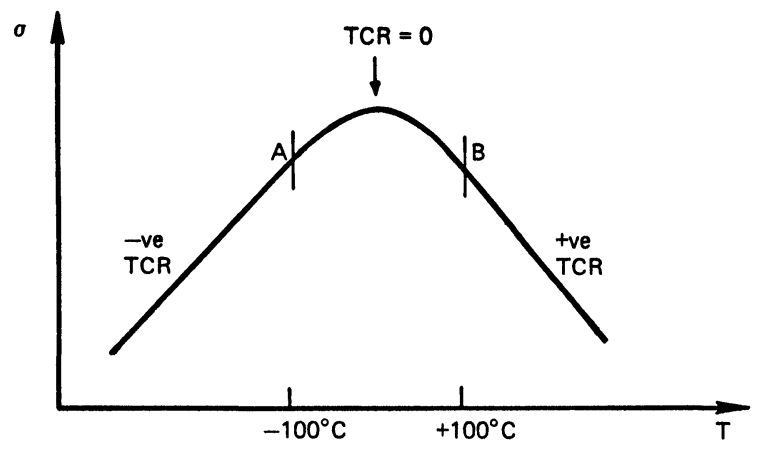

FIGURE 4 Conductivity v. temperature for ideal resistor material. activated process and thus implies a negative TCR. Thus over some low temperature range, in a mixed phase TFR, a negative TCR will be found for conduction, whereas at higher temperatures a positive TCR process will predominate. Conductivity plotted against temperature will give a general characteristic as shown in Figure 4 which demonstrates the transition from negative to positive TCR with increasing temperature.

In order to keep TCRs low and constant over the temperature range in which the TFR is to be used, (say $-100^{\circ} \mathrm{C}$ to $+100^{\circ} \mathrm{C}$ ), the matrix and crystalline material should be selected so that the TCR varies in value about the peak in the conductivity versus temperature plot, that is between A and B in Figure 4 What is more, the range $A$ to $B$ should be as wide as possible to keep the TCR low.

An alternative way of modifying the characteristic A of Figure 3 is by using conducting particles which have a bulk conductivity equal to the highest conductivity component required in the resistor range. By altering the concentration of particles in the matrix material it might be possible to produce a wide range of resistivities by simply varying the number of conduction paths. This situation, characteristic $\mathrm{C}$ of Figure 3, is only possible when a linear variation in the concentration of conducting particles causes a logarithmic change in the resistor conductivity, which appears to be the case for $\mathrm{Bi}_{2} \mathrm{Ru}_{2} \mathrm{O}_{7}$ resistors. This situation seems unlikely, particularly when it is considered that TFRs are designed to be relatively free of electrical noise and insensitive to changes in temperature and to applied pressure. For conducting paths through a system of many particles in contact to be stable, the adhesion between the particles must be strong. The particles should either be locked together by the matrix material, possibly by a slight difference in expansion coefficient, or chemical bonding or sintering should occur between particles at their points of contact. However this situation is still liable to be noisy and unstable and it is more likely for diffusion to take place from the particle into the matrix to reduce the interfacial stress. The matrix would then be doped and could contain sites for conduction with a negative TCR.

\section{WEAKLY THERMALLY-ACTIVATED PROCESSES}

Conduction processes in TFRs will not require strong thermal activation or have a strong dependence on temperature, which rules out processes such as 
Schottky barrier emission, conduction via fieldionisable donor defects, ionic conduction and space charge limited conduction. Processes requiring only a weak thermal activation of less than $\mathrm{kT}$, the thermal vibrational energy of an atom are:

1) Narrow band metallic conduction with dense scattering by imperfections rather than phonons, and

2) Trap tunnelling.

Narrow-band metallic conduction includes the highly positive to nearly zero TCR conduction of simple or ternary oxides. Trap-tunnelling conduction occurs in disordered materials and results in a weak decrease in resistance with increase in temperature Tunnelling of carriers can also occur through a thin insulating matrix between islands of conducting material where the inter-island distance is $<100 \AA{ }^{18}$. But here the process is activated and relatively temperature dependent.

\subsection{Metallic Conduction}

Electrons moving as waves in a metallic crystal interact with ions, limiting the electrons to allowed bands of energy. Electrons in the allowed bands fill an empty crystal until the Fermi level is reached when they are all used up. If the band containing the Fermi level is only partially full, then electrons are free to move in an energy range of the order of $4 \mathrm{kT}$ about the Fermi level. All others are blocked from participation in transport phenomena by the exclusion principle. Mobile electrons in a perfect crystal would have an unlimited mean free path. However no crystal is perfect and electrons travelling through a crystal under the influence of an applied field are impeded by scattering processes arising from lattice vibrations, impurities, defects and other carriers.

At high temperatures scattering of electrons is proportional to the square of the amplitude of the fluctuation of the ions about their equilibrium sites. At temperatures close to absolute zero the metal resistivity reduces to a residual impurity value as electrons are scattered, independently of temperature, by imperfections such as foreign atoms, vacancies, dislocations, grain boundaries and external surfaces. Usually TCRs are $>1000 \times 10^{-6} \mathrm{~K}^{-1}$ but can be reduced by very large densities of defects and impurities. Matthiessen's rule (1862) states that the total resistivity, $\rho$, of a metal is given by

$$
\rho=\rho_{\mathrm{L}}(\mathrm{T})+\rho_{\mathrm{R}}(\mathrm{C})
$$

where $\rho_{\mathrm{L}}$ is a function of temperature and $\rho_{\mathrm{R}}$ a function of the impurity concentration $\mathrm{C}$.
Now if a partially filled band containing the Fermi level covers a small total range of energy the density of states has a large average value over the narrow energy range of the band and the electron effective mass $\mathrm{m}^{*}$ is high ${ }^{22}$. For these heavy electrons the acceleration and mobility $\mu$ in an applied electric field is small

$$
\mu=\frac{\mathrm{e} \tau}{\mathrm{m}^{*}}
$$

where $\tau$ is the relaxation time, which may be the interval between successive collisions in the crystal or the time between electron-phonon transitions. At low temperatures impurity scattering will predominate over scattering by low energy phonons. At higher temperatures the resistivity will increase due to scattering by vibrating ions, but the relative increase will be far smaller than for low mass, high mobility electrons moving under the influence of the same applied field in a wide band material, because of the limitations already imposed by a short mean free path. Thus a low positive TCR might well be expected for a material with a narrow energy conduction band and a suitable large density of impurities.

\subsection{Conduction in Disordered Materials}

The conductivity of liquid metals only drops by a factor of 1.5 to 2.0 on melting ${ }^{23}$ so the metallic nature of conduction and some band structure is retained after melting. Thus long-range order is not essential for metallic conduction.

In disordered materials such as liquid metals and glasses, density of states diagrams have been postulated to be similar to those for crystalline solids except that the energy band states will be smeared out into regions of localised states ${ }^{24}$ as shown in Figure 5.

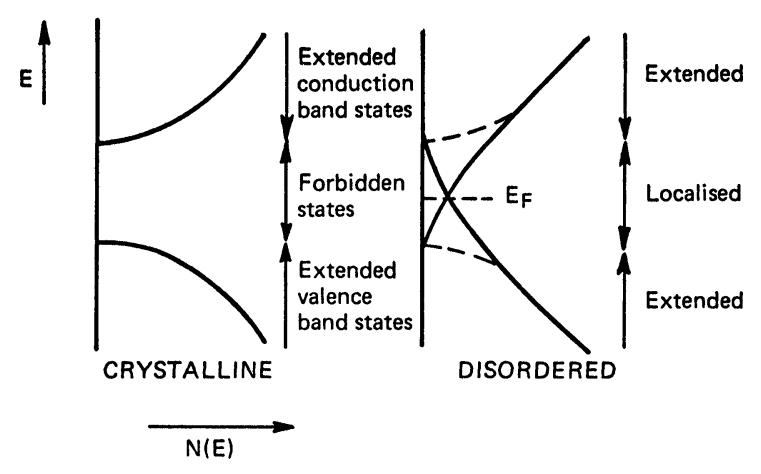

FIGURE 5 The distribution of states in crystalline and disordered solids. 
If the Fermi level lies between the conduction band and valence band states, that is to say that conduction cannot be metallic or narrow band metallic there are two possible mechanisms for conduction via the localised states. These are,

1) Thermal ionisation of carriers, e.g. electrons from donor states, into the conduction band.

2) Phonon-assisted quantum-mechanical traptunnelling or 'hopping' between localised sites.

The first process is a highly negative TCR process and is discounted for TFRs. Trap-tunnelling however is a weakly-activated process and is considered here in more detail.

\subsection{Trap-Tunnelling Conduction}

Localised trap-tunnelling sites are neutral when empty and are thus band-like states. No coulombic field exists which acts against the removal of a carrier. It is expected that the density of trap-like defects would be large at the edge of an extended states region due to the smearing out of the crystalline-type bands. The trapping sites are characterised by an s-like wave function, Figure $6 \mathrm{a}$, and by a shielding potential field of small radius, Figure $6 \mathrm{~b}$. If several trap sites below the conduction band are considered, Figure 7, it can be seen that two conduction paths are possible for transfer of charge from trap A to B. These paths are for,

1) Thermal ionisation and band conduction.

2) Phonon-assisted hopping. ship

The hopping conductivity, $\sigma_{\mathrm{h}}$, obeys the relation-

$$
\sigma_{\mathrm{h}} \propto \exp -(\mathrm{E} / \mathrm{kT}+2 \alpha \mathrm{R})
$$

where $1 / \alpha$ is the decay distance of the localised state, as shown in Figure 6a. A large density of sites is

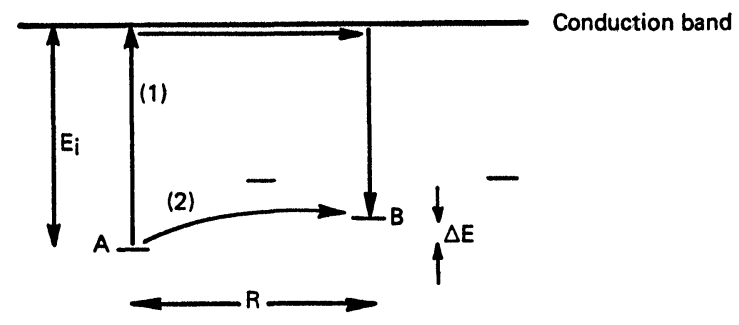

(1) THERMAL IONISATION AND BAND CONDUCTION

(2) PHONON- ASSISTED HOPPING

FIGURE 7 Charge transfer via traps.

required for localised trap-tunnelling and the intertrap distance $\mathrm{R}$ needs to be of the same order as the decay distance. For values of $E>0.5 \mathrm{eV}$ thermal ionisation of carriers into the conduction band is more likely to happen.

The insulating material in TFRs is a glass, a disordered and probably doped material, which may well contain a very large density of localised traps. Mott ${ }^{24}$ considered the case of a constant density of localised states through which the Fermi level passes and derived the relationship,

$$
\sigma_{\mathrm{h}}=\sigma_{0} \exp -\left(A / T^{1 / 4}\right),
$$

where $\sigma_{0}$ is essentially temperature independent and A is a constant dependent on $\alpha$ and the density of trap states. If hopping conduction occurs according to this relationship, then,

$$
\begin{aligned}
\mathrm{TCR} & =-\frac{1}{\sigma} \frac{\partial \sigma}{\partial T}=-\frac{\partial \ln \sigma}{\partial T}=\frac{\partial}{\partial T}\left(\ln \sigma_{0}-\frac{A}{T^{1 / 4}}\right) \\
& =-\frac{1}{4} \frac{A}{T^{5 / 4}}
\end{aligned}
$$

For a TCR of $<100 \times 10^{-6} \mathrm{~K}^{-1}$, acceptable for TFRs, $A$ must be $<0.5 \mathrm{~K}^{1 / 4}$ at $T=300 \mathrm{~K}$. Hill ${ }^{25}$

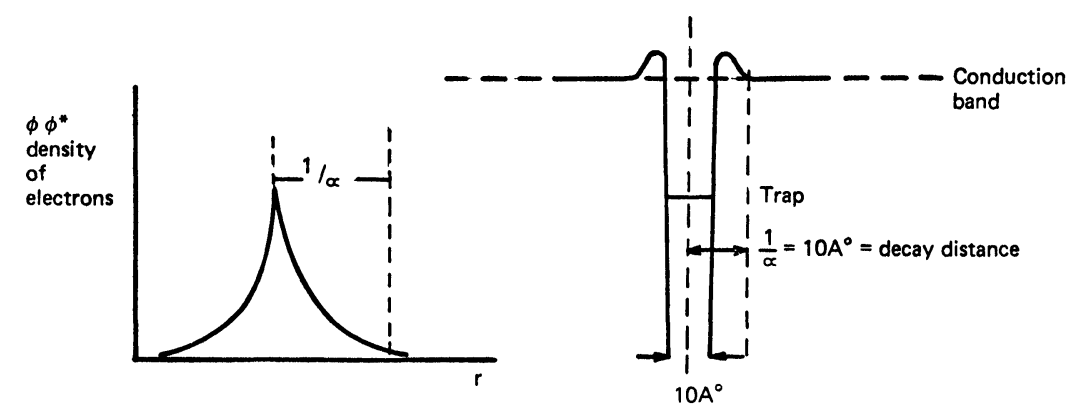

FIGURE 6 (a) Wave function diagram.

(b) Potential diagram for a trap. 
derives an expression for $A$ where,

$$
A=2.31\left(\frac{2 \alpha^{3}}{\pi N_{\mathrm{T}} k}\right)^{1 / 4}
$$

and where $N_{\mathrm{T}}$ is the density of trap sites in units of $\mathrm{cm}^{-3} \mathrm{eV}^{-1}$.

For $A=0.5 \mathrm{~K}^{1 / 4}$ and $\alpha^{-1}=10 \AA$ or $\alpha=10^{7} \mathrm{~cm}^{-1}$, $N_{\mathrm{T}}=3.38 \times 10^{27} \mathrm{~cm}^{-3} \mathrm{eV}^{-1}$ which is far too high for localised states and would represent a sea of metallic states.

For a density of states $N_{\mathrm{T}}=10^{18} \mathrm{~cm}^{-3} \mathrm{eV}^{-1}$, which is an acceptable density for traps with no wave function overlap, $A=120.5$ which at $300 \mathrm{~K}$ corresponds to a TCR of $-24,100 \times 10^{-6} \mathrm{~K}^{-1}$. The TCR is far too high and thus the Mott relationship cannot describe conduction in low TCR TFRs if charge transport is via trap sites.

Hill $^{26}$ considers the passage of charge carriers via a random array of trap sites, of uniform density $\rho_{1}$ $\mathrm{cm}^{-3}$ in real space, which are localised in space and weakly localised in energy. It is assumed that the hopping sites are confined within an energy band $E_{\mathrm{m}} \leqslant k T$ for relative temperature insensitivity. A uniform probability density of carriers $\rho_{2}$ per unit energy exists such that $\rho_{2}=E_{\mathrm{m}}^{-1} \mathrm{eV}^{-1}$. The product $\rho_{1} \rho_{2}$ corresponds to the conventional density of states $N_{\mathrm{T}}$ in units of $\mathrm{cm}^{-3} \mathrm{eV}^{-1}$.

Now the most likely hop within a set of s trap sites will be that to the site of lowest energy difference and lowest radial distance. However the site of nearest radial distance for localised states may not be the site of lowest energy distance, but as one goes out to further neighbours the minimum energy difference decreases. Thus the range of a hop is variable over sites from one to $s$ and we have variable range hopping which is dependent on the excitation energy available. It can also be shown that the most likely transition is one where the supply site and the capture site are equidistant from the Fermi level, so carriers about this level are more mobile.

Assuming $E_{\mathrm{m}} \leqslant k T$ Hill derives expressions for the conductivity and activation energy for resistive and higher conducting material with differing densities of trap sites. A low temperature weak density situation limits hopping to close neighbours with a relatively high activation energy equal to the average difference between sites. Conversely at higher temperatures, with the sites almost de-localised in space, the resistivity becomes proportional to temperature and metallic properties prevail. If the width of the bands is $<k T$, however, the temperature dependence becomes very weak. At room temperature for

$$
\begin{aligned}
& E_{\mathrm{m}} / k T=10^{-1}, \\
& \text { the TCR }=-\frac{E}{k T^{2}}=-300 \times 10^{-6} \mathrm{~K}^{-1},
\end{aligned}
$$

which is appropriate to TFRs.

Hence at the interface between the two extremes of metallic and semi-conducting behaviour in a narrow band of localised states, there will be a mixture of weakly activated and metallic conduction. This transition region corresponds to the region $\mathrm{AB}$ of Figure 4 where the TCR is very small and changes in sign from negative to positive with increase in temperature.

In conclusion charge transfer limiting processes in metallically conducting materials which involve scattering from impurities and disordered atoms have been seen to be temperatures independent in the low temperature regime. Also conduction which takes place in narrow partially-filled bands is far less temperature dependent than usual wide band conduction. Similar conditions hold for trap-tunnelling conduction between trap sites in normally insulating media, where space and energy limitations may allow the hopping process to be only very weakly activated.

\section{ELECTRICAL MEASUREMENTS}

The suggested dual nature of conduction in TFRs may be supported by measuring the activation energies $E_{\mathrm{A}}$ for conduction in commercial resistor systems over a wide temperature range. Running parallel with this work Cash $^{26}$ carried out such measurements on the DuPont 1100, Alloys B and other resistor series. Results for these systems were derived from low d.c. conductivity measurements made from room temperature (RT) down to $4 \mathrm{~K}$. From the Arrhenius equation for activated processes,

$$
\sigma_{\mathrm{T}}=\sigma_{\mathrm{RT}} \exp \left(-\frac{E_{\mathrm{A}}}{k T}\right)
$$

a plot of $\ln \left(\sigma_{\mathrm{T}} / \sigma_{\mathrm{RT}}\right)$ versus $10^{3} / T$ yields a straight line with a slope of $-E_{\mathrm{A}} / k$.

\subsection{Du Pont 1100 Series}

Figure 8 shows the activation plot for resistors 1121 to 1151 from $100 \Omega /$ square to $100 \mathrm{k} \Omega$ /square. A line representing an activation energy of $\mathrm{kT}$ is drawn at the top left of this figure and shows all values of $E_{\mathrm{A}}$ to be much less than $k T$ over the temperature range investigated. Between $10 \mathrm{~K}$ and $30 \mathrm{~K}$ all samples reach a constant activation energy. Resistors 1131 , 


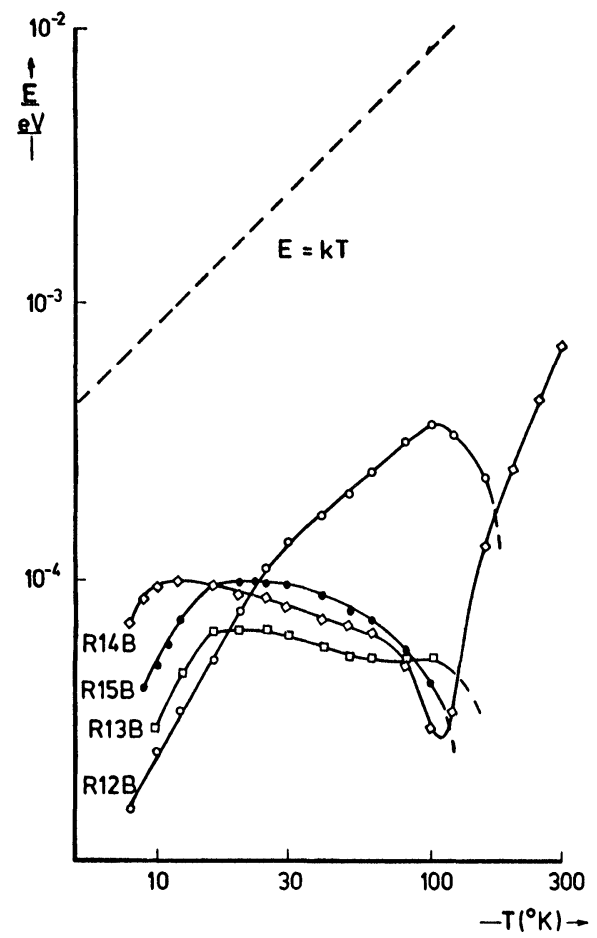

FIGURE 8 Activation energy v. temperature for DuPont 1100 resistors.

1141 and 1151 show clearly defined maxima in $E_{\mathrm{A}}$ as the conduction process changes from a negative activated TCR process to a positive phonon-limited TCR process with increasing temperature.

At low temperatures little thermal energy is available for excitation of carriers and hopping is long range. As the temperature goes up nearer centres become available to hop to and the positive slope, negative TCR region begins with variable range hopping. At the peak in activation energy phonons begin to interact with the carriers as a critical temperature is reached and the carriers begin to become delocalised in energy. With further temperature increase the conductivity becomes narrow band metallic with zero $E_{\mathrm{A}}$ and a phonon-limited mean free path.

The curve for DP1131 differs from the other DuPont resistors in that the narrow band metallic conductivity sets in at a much lower temperature. This behaviour is attributed to the presence of silver particles in the resistor which are apparently required to attain this resistivity value. However, for DP1121, 1141 and 1151 resistors the change in TCR from negative to positive occurs at about room temperature as was previously predicted.

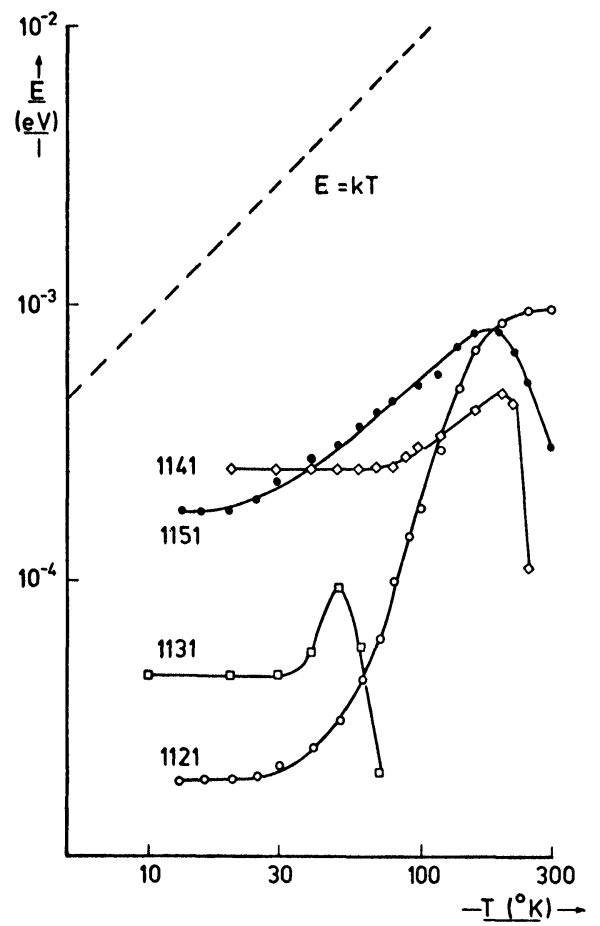

FIGURE 9 Activation energy v. temperature for Alloys B resistors.

\subsection{Alloys B Series}

Maxima in the activation plot, Figure 9, for the Alloys B resistors R12B to R15B are less well defined. The change in sign of the TCR occurs at a lower temperature than for the DuPont resistors and the subsequent decrease in $E_{\mathrm{A}}$ occurs far more slowly. With the change in sign of TCR for the R13B, R14B and $\mathrm{R} 15 \mathrm{~B}$ samples at about $20 \mathrm{~K}$ we expect a sharp fall in activation energy from the analysis of the $E_{\mathrm{A}}$ data for the 1100 series. However, the $E_{\mathrm{A}}$ falls off slowly to about $90 \mathrm{~K}$ when a sharper decline occurs. This behaviour may be ascribed to the doping density of the glass and to the particle sizes which are different from the 1100 series. The dissimilar behaviour of the lower resistance R12B resistor may show an approach to the electrical properties of the bulk oxide particles which are then the major conduction phase.

\section{CONDUCTION IN OBSERVED STRUCTURES}

The micrographs of the DuPont 1100 resistor structures presented in Part I of this paper show the 
conducting particles to be between $0.2 \mu \mathrm{m}$ and $1.0 \mu \mathrm{m}$ in width. Let us consider the number of hypothetically cubic particles of a conducting material which can be packed into a $25 \mu \mathrm{m}$ thick resistor element with the surface dimensions of $1.0 \mathrm{~cm}$ by $0.3 \mathrm{~cm}$. The number of shortest route paths for conduction along the length of the resistor via cube chains is equal to:

\section{1) $1.875 \times 10^{6}$ along $0.2 \mu \mathrm{m}$ width cube chains} only.

2) $7.50 \times 10^{4}$ along $1.0 \mu \mathrm{m}$ width cube chains only.

Here a solid block of the conductor is effectively considered. If however the chains are 'diluted' by an intermediate glassy phase and poor physical and electrical contact between particulate cubes is allowed for, we can establish the resistivity of the conducting chains of fixed particle size required to give an element of a desired resistance value.

For example, the resistivity of the conducting cubes for a resistor of $10 \Omega$ is calculated. Contact between cube faces is assumed to be by only one quarter of the cube face area. For $0.2 \mu \mathrm{m}$ width cubes with inter-cube contact between areas of $1.0 \times 10^{-10} \mathrm{~cm}^{2}$ along $1.875 \times 10^{6}$ chains, with no intermediate glass, the resistivity of the conducting cubes is required to be $1.875 \times 10^{-3} \Omega \mathrm{cm}$. This value is close to that of bulk polycrystalline $\mathrm{Bi}_{2} \mathrm{Ru}_{2} \mathrm{O}_{7}$ which has a resistivity of $8 \times 10^{-4} \Omega \mathrm{cm}$. In fact for this model fewer conducting chains of $\mathrm{Bi}_{2} \mathrm{Ru}_{2} \mathrm{O}_{7}$ are required for a $10 \Omega$ resistor element or alternatively the area of inter-cube contact could be reduced.

For a resistance of $1 \mathrm{M} \Omega, 10^{-5}$ as many $0.2 \mu \mathrm{m}$ chains are required. That is 18.75 chains separated by a high resistivity glass or alternatively longer, more devious chains, which is expected from observation of the DuPont micrographs.

For $1.0 \mu \mathrm{m}$ width cubes, with again inter-cube contact restricted to one quarter of the surface area, only 0.75 paths, or several more devious paths of greater than $1.0 \mathrm{~cm}$ in length, are required to produce a $1 \mathrm{M} \Omega$ resistor.

The DuPont micrographs show a range of particle sizes in contact. Here the controlling cross-section for conduction is the area of particle to particle contact for the smallest particles. As the larger particles represent a lower resistance path, the total length of the conduction path from one end of the resistor to the other is effectively shortened.

TFR manufacturers quote the power dissipation of their resistors to be $\geqslant 4 \mathrm{~W} \mathrm{~cm}^{-2}$. Particulate chain resistor elements described in the previous model, with good contact between particles, would be capable of dissipating $4 \mathrm{~W} \mathrm{~cm}^{-2}$ except for the very highest resistivity composite elements. Power ratings quoted for high resistivity TFRs are however lower than for low resistivity material which is to be expected.

Thus for the DuPont $1100 \mathrm{Bi}_{2} \mathrm{Ru}_{2} \mathrm{O}_{7}$ system a simple low TCR metallic conduction model via particles in good contact is seen to be reasonable, especially as $\mathrm{Bi}_{2} \mathrm{Ru}_{2} \mathrm{O}_{7}$ has a resistivity of about $8 \times 10^{-4} \Omega \mathrm{cm}$ compared with 1.5 to $150 \times 10^{-6} \Omega$ $\mathrm{cm}$ for metals. Here the role of the glass is as a high resistivity supporting matrix only.

This model does, however, assume that contact between particles is firm with preferably a chemical bond between them to eliminate contact resistance. In fact the DuPont micrographs of Part I show that for resistors of $>1 \mathrm{k} \Omega /$ square resistivity, particles appear to have rather less than a quarter of the surface area of a crystalline face in good contact with their nearest neighbours. Filamentary currents calculated for maximum resistor power dissipations in high resistance material are also very high. It is therefore probable that an alternative means of charge transport takes place between particles through thin glass dielectric barriers. Such a conduction process with a small negative TCR would also explain why resistor TCRs are not consistently positive.

Weakly-activated tunnelling via trap sites has been described as such an alternative process. Tunnelling through a high dielectric constant medium may occur via traps spaced $\sim 10 \AA$ apart over a maximum distance of $\sim 100 \AA$ or directly between metallic particles separated by up to $\sim 10 \AA$.

The distribution of multi-size particles on the surfaces of 1141,1151 , and 1161 resistors for equal area samples has been analysed. The number of particles with nearest neighbour separations (NNS) between 0 and $500 \AA$ have been counted for each material. The data is presented in Table I.

As the sample resistivity increases the percentage of particles in the NNS range, 0 to $500 \AA$ decreases. Also the number of particles in close contact within this range is successively reduced from resistor 1141 to resistor 1161 . However as only two-dimensional

TABLE I

\begin{tabular}{|c|c|c|c|}
\hline Resistor & $\begin{array}{l}\text { Total number of } \\
\text { particles in each } \\
\text { equi-area sample }\end{array}$ & $\begin{array}{l}\text { Number of particles } \\
\text { in range NNS } \\
0 \text { to } 500 \AA\end{array}$ & $\begin{array}{l}\text { Percentage } \\
\text { NNS } \\
0 \text { to } 500 \AA\end{array}$ \\
\hline 1141 & 163 & 128 & 78.5 \\
\hline 1151 & 99 & 42 & 42.4 \\
\hline 1161 & 62 & 13 & 21.0 \\
\hline
\end{tabular}


sections were considered, it is almost certain that chains of closely-spaced ( 0 to $500 \AA$ apart) particles will exist in the bulk of 1161 resistors in three dimensions.

Thus it is seen that for the higher resistivity DuPont resistors a charge tunnelling process via traps in a dielectric medium or directly between metallictype particles over $10 \AA$ distances is very likely to occur if the glass contains trapping centres. The magnitude of such a tunnelling current between particles in DuPont 1100 resistors assuming the presence of traps is now calculated.

Let us consider the magnitude of the current due to the passage of charge via trapping sites in a unit $\mathrm{cm}$ cube of a glass. It will be assumed that the charge has energy in a range $\pm k T$ about the Fermi energy level.

The standard relationship for conductivity

$$
\sigma=\text { ne } \mu
$$

where

$$
\begin{aligned}
& \mu=\text { the mobility }\left(\mathrm{cm}^{2} \mathrm{~V}^{-1} \mathrm{~s}^{-1}\right) \\
& \mathrm{n}=\text { the number of carriers }\left(\mathrm{cm}^{-3}\right)
\end{aligned}
$$

can be written,

$$
J=\operatorname{ne} \mu F
$$

where

$$
\begin{aligned}
& J=\text { current per unit area }\left(A \mathrm{~cm}^{-1}\right) \\
& F=\text { applied field }\left(V \mathrm{~cm}^{-1}\right)
\end{aligned}
$$

For the energy range we consider

$$
J=\left(N_{\mathrm{t}} 2 k T\right) \mathrm{e} \mu F
$$

where $\mathrm{N}_{\mathrm{t}}$ is the density of states $\left(\mathrm{cm}^{-3}(\mathrm{eV})^{-1}\right)$.

By Einstein's relationship for the diffusion coefficient $D\left(\mathrm{~cm}^{2} \mathrm{~s}^{-1}\right)$,

$$
D=\frac{\mu k T}{e}
$$

Also

$$
D=\frac{R^{2}}{z} \nu_{\mathrm{ph}} p_{\mathrm{R}}
$$

from a consideration of the number of nearest neighbours, $z$, where $R=$ inter-trap distance $(\mathrm{cm})$

$$
\begin{aligned}
& \nu_{\mathrm{ph}}=\text { phonon frequency }\left(\mathrm{s}^{-1}\right) \\
& p_{\mathrm{R}}=\text { probability of a hop between sites. }
\end{aligned}
$$

Therefore

$$
\mu=\frac{e}{k T} \frac{R^{2}}{z} \nu_{\mathrm{ph}} p_{\mathrm{R}}
$$

From Eqs. (3) and (6)

$$
J=2 \frac{e^{2}}{z} N_{\mathrm{t}} F R^{2} \nu_{\mathrm{ph}} p_{\mathrm{r}}
$$

At $300 \mathrm{~K}$ the Mott relationship for $p_{\mathrm{R}}$ is invalid for low TCR conduction, as seen before. Here Hill's conduction theory is applicable. However, if $N=$ $10^{18} \mathrm{~cm}^{-3} \mathrm{eV}^{-1}$ and the probability term is taken to equal 0.5 the hopping current can be calculated by using equation (7) and the values,

$$
\begin{aligned}
e & =1.6 \times 10^{-19} \text { coulombs } \\
F & =5 \mathrm{~V} \mathrm{~cm}^{-1} \\
R & =10 \AA=10^{-7} \mathrm{~cm} \\
\nu_{\mathrm{ph}} & =10^{12} \mathrm{~s}^{-1} \\
z & =6 .
\end{aligned}
$$

Dividing Eq. (7) by a factor of $1.6 \times 10^{-19}$ to make the units of $e$ and $N_{\mathrm{t}}$ compatible,

$$
J=2.7 \times 10^{-4} \mathrm{~A} \mathrm{~cm}^{-1}
$$

and for a unit $\mathrm{cm}$ cube the resistance,

$$
R=1.85 \times 10^{4} \Omega
$$

and the resistivity,

$$
\rho=1.85 \times 10^{4} \Omega \mathrm{cm}
$$

for an applied field of $5 \mathrm{~V} \mathrm{~cm}^{-1}$.

For a high resistivity TFR of dimensions $1 \mathrm{~cm} \mathrm{x}$ $1 \mathrm{~cm} \times 20 \mu \mathrm{m}$ thick, comprised of the glassy phase only containing trap sites, and a bulk resistivity of $1.85 \times 10^{4} \Omega \mathrm{cm}$, the resistance,

$$
R=\frac{\rho L}{A}=\sim 10^{7} \Omega
$$

Thus a range of $\Omega$ /square values of between $10 \Omega /$ square and $\sim 1 \mathrm{M} \Omega$ /square can be obtained by suitable mixing of the two phases. Such components would dissipate power more evenly throughout their bulk than the filamentary model resistors discussed earlier which considered the glassy phase to take no part in conduction. It is of note in the combined conduction model that for the narrow band of hopping sites assumed for the glass and for the narrow partially-filled conduction band proposed for the crystalline phase, the same restrictions are imposed on the carrier energies in both cases and thus energy continuity can occur at the phase boundaries.

A similar dual conduction model can be argued for the Alloys $\mathrm{B} \mathrm{RuO}_{2}$ system and the Electroscience $7000 \mathrm{Pd} / \mathrm{PdO} / \mathrm{Ag}$ system, assuming that the microcrystalline material has a low TCR. Here the crystallite sizes are smaller, of the order of $0.25 \mu \mathrm{m}$, and 
the particles are dispersed more evenly in the glass matrix. Control of the resistivity by varying the glass to oxide ratio is thus improved, and this may offset the less electrically stable properties of the particle phase, when compared with $\mathrm{Bi}_{2} \mathrm{Ru}_{2} \mathrm{O}_{7}$. In accordance with this hypothesis, later DuPont systems are known to have a range of smaller $\mathrm{Bi}_{2} \mathrm{Ru}_{2} \mathrm{O}_{7}$ particle sizes ${ }^{2}$ than the 1100 series.

$\mathrm{PbO}-\mathrm{RuO}_{2}$ solid solutions are known to form ${ }^{2} 8$ of lower TCR than $\mathrm{RuO}_{2}$ alone and if this occurs in the Alloys B resistors, the two phases should be more energetically compatible. Oxidising and reducing reactions in $\mathrm{Pd} / \mathrm{PdO} / \mathrm{Ag}$ systems are known to be complex and dependent on the TFR firing process.

In the three systems considered trap sites may be formed either at nucleation centres in the glass, or by incomplete bonding in the glass matrix, or by diffusion of metal ions into the glass.

\section{CONCLUSIONS}

From experimental observations it has been seen that TFRs are principally two phase materials, with a doped lead borosilicate glass surrounding crystalline particles. The phases have been identified for each resistor series as follows:

DuPont 1100: $\quad \mathrm{Bi}_{2} \mathrm{Ru}_{2} \mathrm{O}_{7}$ crystallites and a $\mathrm{CdO}$ doped glass.

Alloys B: $\quad \mathrm{RuO}_{2}$ crystallites and a $\mathrm{Ru}$, Ag doped glass.

Electroscience 7000: $\mathrm{Pd} / \mathrm{PdO} / \mathrm{Ag}$ crystallites and a $\mathrm{Pd}, \mathrm{Ag}$ doped glass.

Resistor systems have family properties where an increase in crystallite content raises the conductivity. Resistor TCRs change sign from negative to positive as the temperature rises between $10 \mathrm{~K}$ and $300 \mathrm{~K}$. TCRs are also low in a temperature range about room temperature.

These properties are a result of the following factors. A mixed conduction process takes place which is a combination of the processes occurring in the two phases. The crystallite phases conduct metallically, In the case of $\mathrm{Bi}_{2} \mathrm{Ru}_{2} \mathrm{O}_{7}$ the resistivity is only $\sim 10^{-4} \Omega \mathrm{cm}$, due to conduction occurring in a narrow partially-filled band, which implies high effective mass carriers. Charge flow through the glassy phase occurs by trap-tunnelling with low activation energies between $10^{-5}$ to $10^{-3} \mathrm{eV}$. This can only happen in a narrow energy band of states. This narrow band is formed by the doping of the glass by ions either incorporated in the glass structure or diffused in from the crystalline phase. Resistors are stable because the properties imparted during the firing process are locked into the glass when it cools to room temperature.

\section{ACKNOWLEDGEMENTS}

This work was carried out with the support of Procurement Executive, Ministry of Defence at the Dept. of Physics, Chelsea College, London. Permission from the MoD to publish this work is gratefully acknowledged.

\section{REFERENCES}

1. L. C. Hoffman, Amer. Cer. Soc. Bull., 42, 490 (1963).

2. H. C. Angus and P. E. Gainsbury, Electronic Components, 9, 84 (1968).

3. G. S. Iles, Radio and Electronic Engineer, 11, 299 (1968).

4. T. H. Lemon, Chemistry and Industry, 19, 920 (1973).

5. P. R. van Loan, Insulation/Circuits, 6, 35 (1972).

6. W. D Ryan et al., Phys. Rev. B, 1, 1494 (1970)

7. D. B. Rogers et al., Inorganic Synthesis (McGraw-Hill 1972. Ed. F. A. Cotton) Vol. XIII, p. 135.

8. S. R. Butler and J. L. Gillson, Mat. Res. Bull., 6, 81 (1971).

9. R. J. Bouchard, U.S. Patent No. 3,583,931.

10. M. V. Coleman and P. W. Graves, 1972. Third Report RDRDC Contract No. K27A/486/CB27A/2.

11. R. J. Bouchard and J. L. Gillson, Mat. Res. Bull., 6, 669 (1971).

12. A. W. Sleight and R. J. Bouchard, Nat. Bur. Stds., Special Publication No. 364, Solid State Chemistry 227 (1972).

13. R. F. Geller and E. N. Bunting, J. Res. Nat. Bur. Stds., 23, 275 (1939).

14. W. M. Faber et al., U.S. Patent No. 3,304,199.

15. C. L. Holmes, U.S. Patent No. 3,324,049.

16. G. L. Fuller, Microstructure and Mechs. of Elec. Cond. in $\mathrm{RuO}_{2}$-Glass TFRs. Ph.D. Thesis, Purdue University (1971).

17. R. C. Buchanan and M. A. Zuegel, J. Amer. Cer. Soc., 51, (1968).

18. R. M. Hill, Proc. Roy. Soc. A., 309, 377 (1969).

19. A. Malliaris and D. T. Turner, J. Appl. Phys., 42, 614 (1971).

20. T. E. Christen and J. G. Hewitt, Elec. Comps. Conf., USA (1970).

21. A. Matthiessen, Rep. Brit. Ass., 32, 144 (1862).

22. J. S. Blakemore, Solid State Physics (W. B. Saunders and Co. 1969).

23. A. I. Gubanov, Quantum Electron Theory of Amorphous Conductors (Consultants Bureau, 1965).

24. N. F. Mott and E. A. Davis, Electronic Processes in NonCrystalline Materials (Clarendon Press, Oxford, 1971).

25. R. M. Hill, Phil. Mag., 23, 59 (1971).

26. D. A. Cash, M. P. Ansell and R. M. Hill, 1974. Final Report MoD Contract No. RU7-41.

27. M. V. Coleman, Private Communication, 1975.

28. T. Kasanami et al., U.S. Patent No. 3,778, 389. 

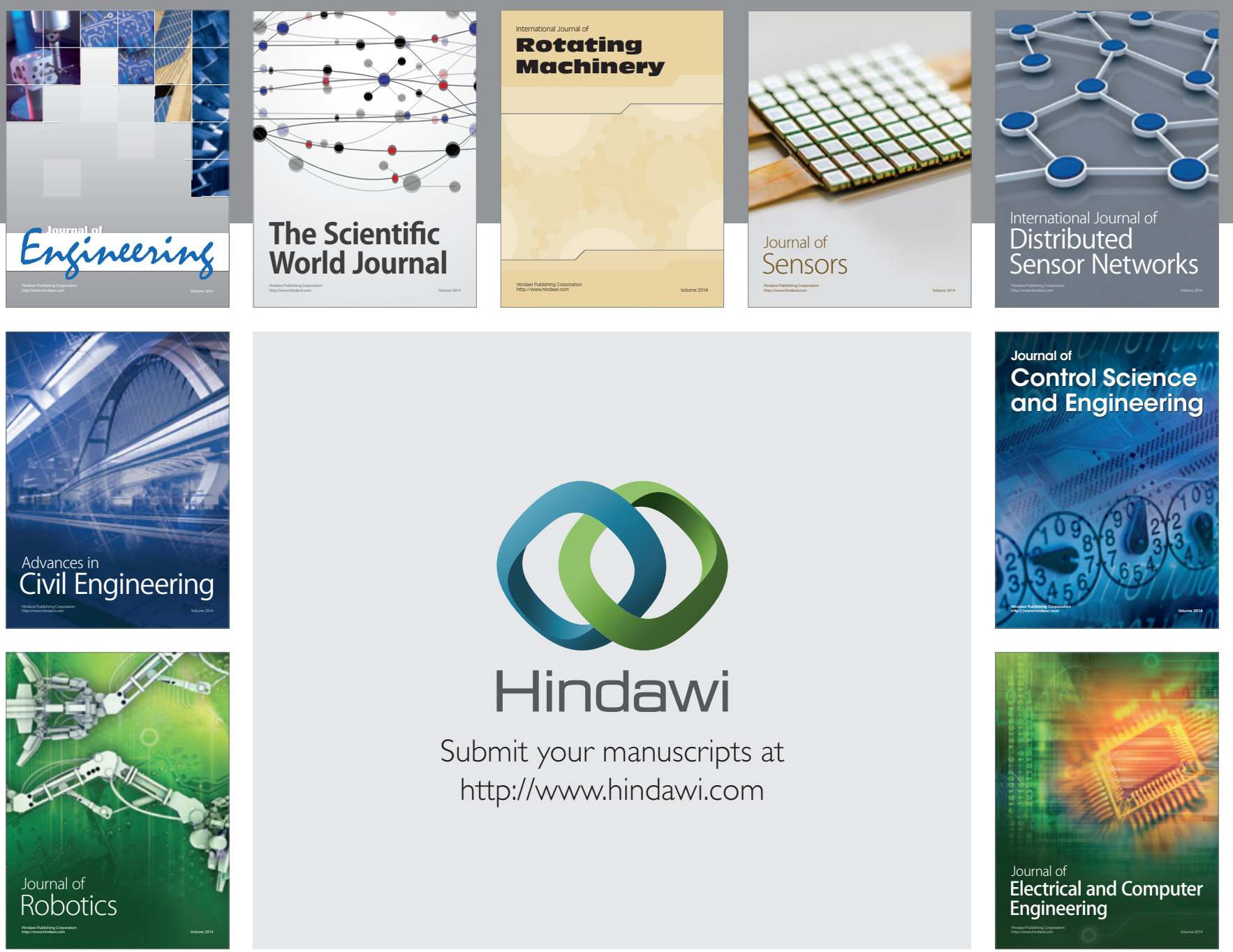

Submit your manuscripts at

http://www.hindawi.com
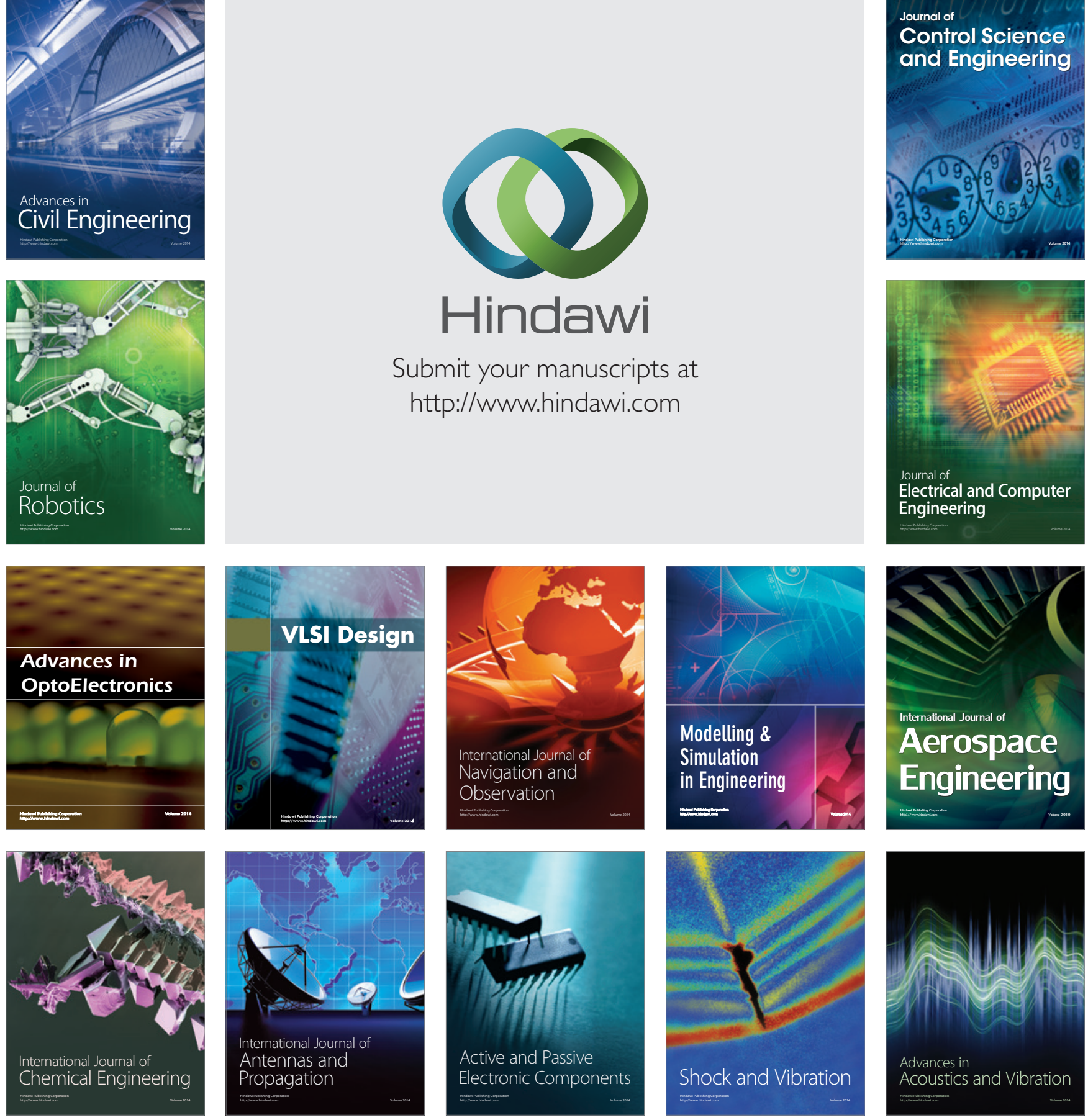